Check for updates

Cite this: RSC Adv., 2019, 9, 38547

Received 8th October 2019

Accepted 20th November 2019

DOI: $10.1039 / c 9 r a 08178 j$

rsc.li/rsc-advances

\title{
Reduced graphene oxide-metal nanoparticle composite membranes for environmental separation and chloro-organic remediation $\uparrow$
}

\author{
Ashish Aher, ${ }^{a}$ Samuel Thompson, ${ }^{a}$ Trisha Nickerson, ${ }^{a}$ Lindell Ormsbee ${ }^{b}$ \\ and Dibakar Bhattacharyya (iD) *a
}

This study explores the integration of separation performance of rGO membrane with heterogeneous oxidation reactions for remediation of organic contaminants from water. Herein, an approach was introduced based on layer-by-layer assembly for functionalizing rGO membranes with polyacrylic acid and then by in situ synthesis of Fe based reactive nanoparticles. TEM characterization of the crosssection lamella of the membranes showed a high density of nanoparticles ( $12 \% \mathrm{Fe}$ ) in the functionalized domain, signifying the importance of polyacrylic acid for in situ synthesis of nanoparticles. The membranes exhibited a pure water permeability of $1.9 \mathrm{LMH}$ bar $^{-1}$. The membranes had low to moderate salt retention, and more than $90 \%$ neutral red retention (organic probe molecule, size: $1.2 \mathrm{~nm}$ ). The membranes also exhibited high retention of humic acids (80\%), preventing these organics from entering the reactive domain, and thus potentially reducing the formation of undesired by-products. A persulfate mediated oxidative pathway was employed to demonstrate the reactive removal of organic contaminants. The membranes achieved $>95 \%$ conversion by convectively passing $2 \mathrm{mM}$ persulfate feed at a transmembrane pressure of 0.4 bar. Successful degradation of TCE (up to $61 \%$ ) was achieved in a single pass by convective flowing of the feed solution through the membrane, generating up to $80 \%$ of the theoretical maximum chloride as one of the byproducts. Elevated temperatures significantly enhanced persulfate mediated TCE oxidation extent from $24 \%$ at $23{ }^{\circ} \mathrm{C}$ to $54 \%$ at $40{ }^{\circ} \mathrm{C}$ under batch operating conditions.

\section{Introduction}

Membrane-based separation technologies are playing a pivotal role in addressing the challenge of the increasing need for potable water by removing organic contaminants via different exclusion mechanisms. ${ }^{1-7}$ In addition to the applications in separation, a membrane platform can be utilized as a support for embedding reactive nanoparticles. Reactive membranes provide an opportunity to conduct reactions, such as heterogeneous oxidation, in a continuous mode of operation for the removal of organic contaminants. ${ }^{8}$ The immobilization of reactive/catalytic components also eliminates the concerns associated with catalyst recovery. ${ }^{9,10}$ This study explored the integration of membranes with advanced oxidation processes (AOPs) by embedding reactive $\mathrm{Fe} / \mathrm{Pd}$ nanoparticles in reduced Graphene Oxide (rGO) membranes for removal of persistent organic contaminants from water.

${ }^{a}$ Chemicals and Materials Engineering Department, University of Kentucky, 177 FPAT Bldg, Lexington, KY, 40506, USA. E-mail: db@uky.edu; Tel: +1-859-312-7790 ${ }^{b}$ Civil Engineering Department, University of Kentucky, Lexington, KY, 40506, USA $\dagger$ Electronic supplementary information (ESI) available. See DOI: $10.1039 / \mathrm{c} 9 \mathrm{ra} 08178 \mathrm{j}$
rGO membranes have attracted increasing attention of the membrane community for aqueous and organic solvent nanofiltration. ${ }^{11,12}$ Besides nanofiltration applications, rGO tends to participate in electron transfer reactions involved in oxidation reactions..$^{13}$ In oxidation reactions, the electron transfer reactions result in the fission of the peroxy linkage of oxidizing agents, generating free radicals for the destruction of organic contaminants. Active sites involved in electron transfer reactions are believed to be the edge defects present in the basal plane of rGO. ${ }^{14-16}$ Ketonic groups present on the basal plane has higher electronic density, which enhances the tendency of these materials to get involved in the electron transfer reactions. ${ }^{15}$ Doping of GO with nitrogen and sulfur have also been reported to enhance the capability of GO to improve reaction efficiency because of the redistribution of charge densities and creation of more active sites. ${ }^{17-19}$

Furthermore, rGO provides support for the immobilization of reactive components. Nanocomposites of rGO with the transition metals are reported to perform better in AOP. ${ }^{20-22}$ In the nanocomposites, transition metals are used for the initiation of free radical reactions, whereas, rGO serves as a platform for immobilization. Better activation performance for the magnetite-GO composite as compared to magnetite alone in 
the decomposition of trichloroethylene (TCE) through AOP is reported by Ahmad et al. ${ }^{23}$ rGO being hydrophobic, can adsorb organic compounds with aromatic groups through pi-pi stacking, resulting in a synergetic effect of adsorption and surface-initiated radical formation towards organic contaminant degradation. ${ }^{19}$ Successful incorporation of reactive nanoparticles in the rGO membrane, therefore, should permit decomposition of organic impurities in addition to the separation capabilities of rGO membranes.

rGO-based reactive membranes have shown promising results in removal of a wide range of organic contaminants, such as phenols, nitrophenol, dyes, etc. ${ }^{24-26}$ Metal-free GO membranes synthesized by doping basal plane of GO with heterogeneous atoms have shown improved performance in breaking down persulfate to generate free radicals for oxidative degradation of organic contaminants. ${ }^{24}$ The introduction of metal nanoparticles provides an opportunity for conducting peroxy mediated oxidation reactions at a faster rate and room temperatures. Approaches such as surface and nanochannel functionalization are employed to synthesize reactive rGO membrane platforms. Soroush et al. synthesized GO membranes decorated with silver nanoparticles, which exhibited antimicrobial properties. ${ }^{27}$ Incorporation of $\mathrm{TiO}_{2}$ nanoparticles in the nanochannels of GO by co-filtration of nanoparticles and GO was demonstrated by Gao et al. ${ }^{25}$ The membranes showed photocatalytic abilities, reactively removing phenols under the light. Although these approaches have been successful in synthesizing a reactive membrane platform, the remobilization of nanoparticles in the membrane domain in the event of leaching is a challenge. The incorporation of functional polymers in rGO layers introduces ligands in the membrane domain, which could be used towards the immobilization of precursors for nanoparticle synthesis. Subsequent in situ synthesis of nanoparticles is one of the assuring ways for the re-functionalization of the membranes.

Furthermore, free radical-mediated oxidation processes are typically accompanied by the formation of byproducts from side reactions of free radicals with natural organic matter (NOM) present in water. ${ }^{28,29}$ Removal of NOM before oxidation processes is desirable to mitigate the formation of halogenated by-products of these organics. rGO membranes have shown high removal efficiency for NOM, primarily by size exclusion mechanism..$^{30}$ A nanocomposite membrane of rGO with a top layer comprised of rGO provides an opportunity to remove NOM by size and charge exclusion, and the formation of byproducts by oxidation reactions in the reactive domain underneath can be minimized.

The novelty of this study lies in combining the persulfate mediated oxidation reactions with the separation performance of rGO membranes in a loose nanofiltration regime. This study demonstrates the synthesis and application of reactive rGO membranes for the removal of chlorinated organics from water. The objectives of the study were: (1) to incorporate polyacrylic acid in the nanoporous domain of rGO membranes for in situ synthesis of nanoparticles, (2) to establish the role of polyacrylic acid in synthesis of reactive nanoparticles in membrane domain using advanced characterization, (3) to understand impact on flux and separation performance of the rGO membranes after incorporating PAA and reactive nanoparticles, by using dye (probe molecule), salt solutions and humic acids (model compound for NOM), and (4) to investigate persulfate mediated oxidative removal efficiency of trichloroethylene (TCE, model compound) by reactive rGO nanocomposite membrane.

\section{Material and methods}

\subsection{Materials}

GO was obtained as an aqueous dispersion $\left(4 \mathrm{mg} \mathrm{ml}^{-1}\right)$ from Graphenea Inc. All other chemicals used in this study were of reagent grade and were purchased from Fischer Scientific or VWR. Ultrapure water used in all experiments was purified (resistivity $>18 \mathrm{M} \Omega$ ) using a Purelab flex water purifier obtained from ELGA lab water. A commercial-grade polyvinylidene fluoride (PVDF) membrane PV200 from Nanostone Water Inc. was used as a substrate for the synthesis of the GO membranes. The PVDF membrane had a pore size in the range of 90-120 nm and a PVDF thickness of approximately $110 \mu \mathrm{m}$ coated on a nonwoven fabric (ESI Fig. S1†).

\subsection{Synthesis of nanocomposite rGO membranes}

Nanocomposite rGO membranes were synthesized on a PVDF membrane support using a layer-by-layer (LBL) assembly approach, schematically represented in Fig. 1a. First, GO with a loading of $60 \mathrm{mg} \mathrm{m}^{-2}$ was deposited on the PVDF substrate by pressure-assisted filtration. Acrylic acid (5 wt\%) was then polymerized on the surface of GO under UV light in the presence of bisacrylamide (5 mol\% of acrylic acid) as a crosslinker and 1hydroxycyclohexyl phenyl ketone as an initiator. A thin coat of GO (10-15 $\mathrm{mg} \mathrm{m}^{-2}$ ) was then deposited on top of the functionalized GO surface. Five such cycles of UV-initiated polymerization followed by the deposition of a thin layer of GO were carried out to synthesize a nanocomposite layer. The composite membranes were incubated in an oven at $90{ }^{\circ} \mathrm{C}$ for $20 \mathrm{~h}$ to reduce the deposited GO layers. Ferrous ions were then immobilized on the introduced carboxylate ligands of PAA, by ion exchange cycle and were later reduced to zerovalent Fe using $\mathrm{NaBH}_{4}$, as reported in our earlier publication. ${ }^{6}$ For the synthesis of Fe-Pd bimetallic NPs, palladium was coated on the surface of the zerovalent Fe through redox reactions at the Fe surface by contacting membrane with aqueous Pd solution. The schematic representation and optical image of the nanocomposite membranes are shown in Fig. 1b, c, and ESI Fig. S3. $\dagger$

\subsection{Characterization}

Cross-section of the membrane was imaged using transmission electron microscopy (TEM, Talos F200X) coupled with energy dispersive spectroscopy (EDS, Oxford Instrument X-Max ${ }^{\mathrm{N}} 80$ detector). A TEM sample of the nanocomposite rGO layer crosssection was prepared using a focused ion beam (Helios FEI). Xray photoelectron microscopy (XPS, Thermo Scientific K-Alpha) was used to determine the elemental composition across the membrane depth. The crystal structure and the specific surface area of the Fe-based nanoparticles synthesized in the solution 

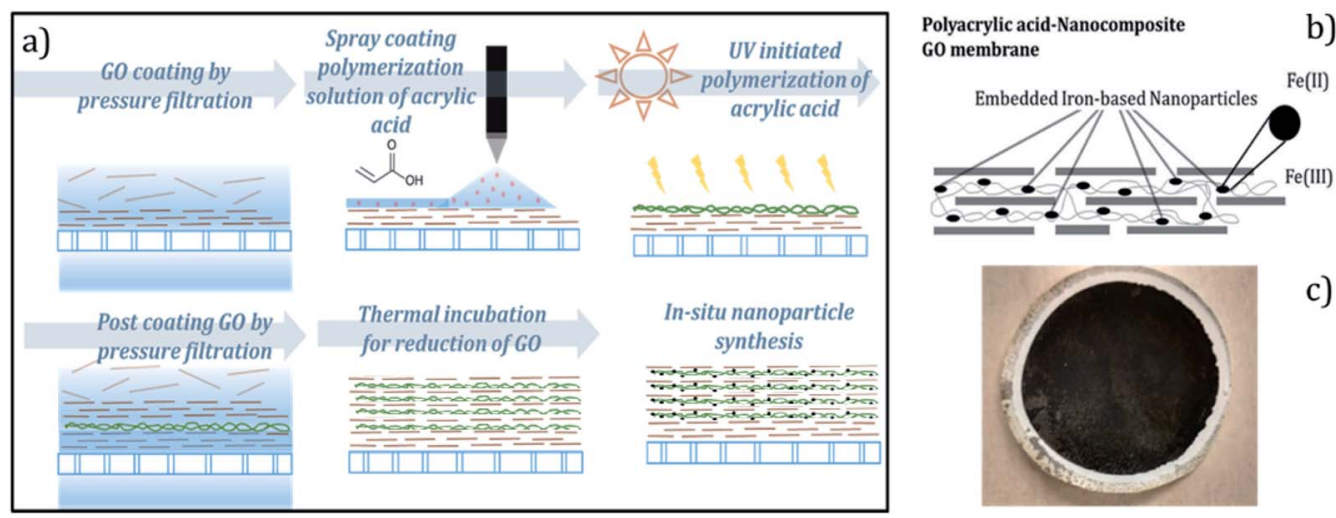

Fig. 1 Schematic representation of (a) membrane synthesis and (b) nanoparticles embedded polyacrylic acid-rGO nanocomposite membranes. (c) Optical image of nanoparticles embedded polyacrylic acid-rGO nanocomposite membrane.

phase were determined using X-Ray Diffraction (XRD, Siemens D500, $\mathrm{Cu} \mathrm{K} \alpha 1.5418 \AA$ ) and a Brunauer-Emmett-Teller (BET, Micromeritics TriStar 300) analyzer. Functional groups of rGO were characterized by Fourier Transform Infrared Spectroscopy (FTIR, Varian 7000e). Contact angles were measured using a Drop shape analyzer (DSA 100 Kruss), and C 1s binding energy spectra were obtained using XPS.

\subsection{Performance analysis}

The performance of the membranes was tested in a dead-end (Sterlitech HP4750) and a cross-flow (Sterlitech CF016D) mode of operation. The experimental set-up is schematically represented in ESI Fig. S2. $\dagger$ Pure water permeability (pressurenormalized flux) of the membranes was determined for transmembrane pressure (TMP) up to 10 bar. The percentage rejections of species by the membranes were determined using eqn (1).

$$
\text { Rejection }(\%)=\left(1-\frac{C \_ \text {perm }}{C \_ \text {feed }}\right) \times 100
$$

where, $C_{-}$feed, $C_{-}$perm, and $C_{-}$ret are the concentrations of feed, permeate and retentate, respectively.

Reactivity of the membrane was assessed by studying persulfate decomposition. The decomposition of persulfate $(2 \mathrm{mM})$ by nanocomposite rGO membranes was measured in a deadend mode of operation under different TMPs. Control tests were conducted with blank rGO membranes without nanoparticles. Oxidation of trichloroethylene (TCE) by the nanocomposite rGO membrane was also conducted in a dead-end mode of operation in the presence of $2 \mathrm{mM}$ persulfate at $\mathrm{pH} 7$.

\subsection{Analysis}

Persulfate concentration was measured using iodometric titrations. ${ }^{31}$ TCE was analyzed by Gas Chromatography (Varian CP3800)-mass spectrometry (Saturn 2200) instrument with helium as a carrier gas. Chloride concentrations were measured as an additional check for the oxidation of TCE using a chloride probe (Acumet). Analysis of the intermediates of TCE oxidation was done by GC-MS analysis after the derivatization of the reaction intermediates. For derivatization, the intermediates were esterified with hexyl alcohol by conducting a reaction of $10 \mathrm{ml}$ of solution with $500 \mathrm{ppm}$ hexyl alcohol at $60{ }^{\circ} \mathrm{C}$ for 20 minutes in the presence of $\mathrm{H}_{2} \mathrm{SO}_{4}$ as a catalyst.

\section{Results and discussion}

The primary goal of the study was to synthesize a reactive rGO membrane platform for the oxidative removal of organic contaminants from water. The current section discusses the approach used in this study for synthesis of the membranes, characterization and performance evaluation, this being followed by a discussion on persulfate mediated oxidation of TCE from aqueous solution in solution phase and in reactive membrane domain (model compound for volatile organic contaminants).

\subsection{Membrane cross-section characterization}

One of the aims of this study was to characterize the crosssection of the nanocomposite membrane to measure the thickness of the nanocomposite layer and to investigate the distribution of nanoparticles across the domain. Determining the distribution of iron across the GO layers was critical to understand the role of polyacrylic acid in in situ nanoparticle synthesis. Also, the residence time of reactants in the membrane domain is directly proportional to the thickness, and therefore, needs to be assessed.

The cross-section morphology of a nanocomposite rGO membrane sample synthesized in the study is shown in Fig. 2a. The membrane surface was coated with platinum before making the lamella to prevent any damage during milling by a Ga beam. The deposited rGO layers exhibited two distinct regions: nanocomposite of rGO-PAA and underneath rGO (Fig. 2b). The observed thickness of the rGO-PAA layer and the underneath rGO layer were 110 and $150 \mathrm{~nm}$, respectively. The high-resolution TEM image showed the presence of nanoparticles in the membrane domain (Fig. 2b). EDS mapping was performed across the different deposited layers to determine their elemental composition. Elemental composition in the 

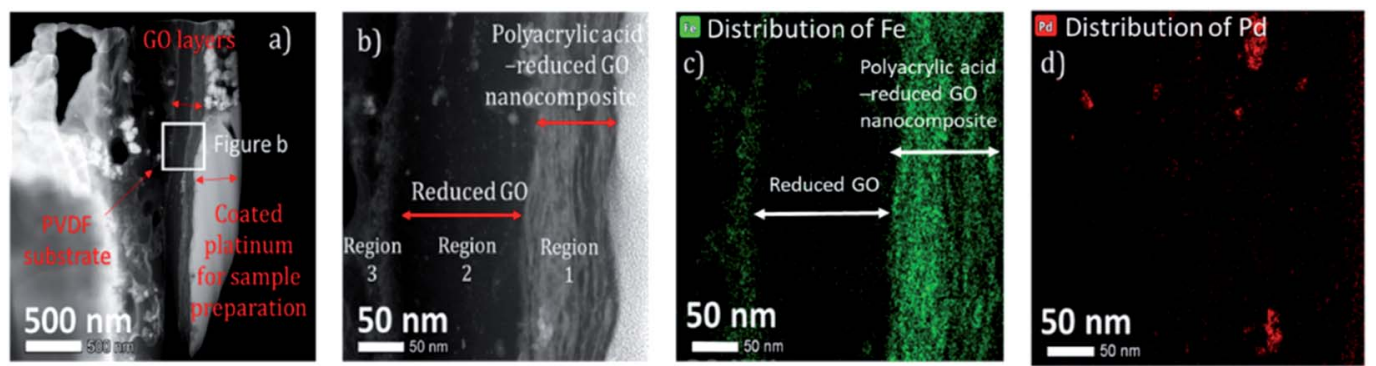

\begin{tabular}{|l|l|l|l|}
\hline e) Composition & $\begin{array}{l}\text { Substrate } \\
\text { (Region 3) }\end{array}$ & $\begin{array}{l}\text { rG0 } \\
\text { (Region2) }\end{array}$ & $\begin{array}{l}\text { Nanocomposite } \\
\text { rG0 (Region 1) }\end{array}$ \\
\hline Carbon (C) & 57 & 75 & 40 \\
\hline Oxygen (0) & 29 & 22 & 48 \\
\hline Fluorine (F) & 10 & 2 & nd \\
\hline Iron (Fe) & 3 & 0.5 & 12 \\
\hline Palladium (Pd) & 0.1 & 0.4 & 0.3 \\
\hline
\end{tabular}

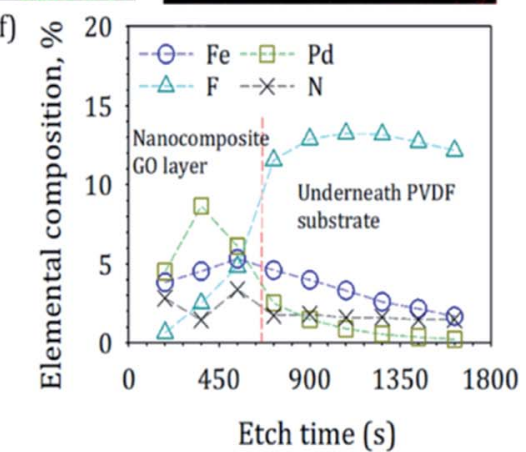

Fig. 2 Cross-section of the nanocomposite membrane imaged using Transmission Electron Microscopy. (a) HAADF image of the lamella of GO membrane cross-section prepared using a focused ion beam. (b) High-resolution image of the nanocomposite membrane showing active later of GO membrane (reduced GO (region 2) and polyacrylic acid composite GO (region 1)). (c and d) Distribution of Fe and Pd across the membrane cross-section obtained using EDS, respectively. (e) Elemental composition of the nanocomposite membranes in the three regions as highlighted in (b). (f) Elemental composition determined across the depth of the membrane using XPS. The remainder of the elemental composition represented in (f) is carbon.

three regions of nanocomposite membranes is summarized in Fig. 2e and EDS spectra for the nanocomposite domain (region 1, Fig. 1b) can be found in ESI Fig. S4. $\dagger$ The rGO-PAA domain had a high density of Fe compared to the underneath rGO domain (Fig. 2c). The Fe concentration dropped from $12 \%$ in the polymer nanocomposite domain to $0.5 \%$ in the rGO domain. Also, a slightly higher concentration of Fe (3\%) was observed in the PVDF substrate, which could be attributed to the more open structure of PVDF domain compared to rGO domain. Owing to the relatively low atomic proportion of $\mathrm{Pd}$, weak signals for Pd were observed across the membrane thickness along with the presence of some agglomerates (Fig. 2d). The high density of nanoparticles in the PAA functionalized rGO domain compared to the nonfunctionalized domain signifies the importance of introducing carboxylate groups for the in situ synthesis of nanoparticles.

Elemental composition across the membrane thickness was also determined using XPS (Fig. 2f). Membrane surface had $56 \% \mathrm{C}, 3 \% \mathrm{~N}, 4 \% \mathrm{Fe}, 32 \% \mathrm{O}$ and $5 \% \mathrm{Pd}$. The membrane sample was etched using a $1000 \mathrm{eV}$ argon beam, and the elemental composition was determined at different etched intervals. Spectra corresponding to the binding energies of the analyzed elements are shown in ESI Fig. S5. $\dagger$ Fluorine concentration increased gradually and reached a maximum of $14 \%$ after an etch time of $900 \mathrm{~s}$. During initial etching cycles, fluorine from the lower PVDF substrate was not observed, suggesting the absence of defects. After etching the membrane sample for $900 \mathrm{~s}$, the argon beam likely had penetrated through the nanocomposite rGO layer, and photoelectrons from the underneath substrate were likely measured by the detector. The oxygen content also declined after $900 \mathrm{~s}$ of etching to $2 \%$. As expected, the elemental proportion of $\mathrm{Fe}$ and $\mathrm{Pd}$ declined beyond $900 \mathrm{~s}$ of etching, as there are no ligands present in the PVDF matrix to immobilize the precursor for nanoparticle synthesis.

Naturally, one may desire to have high metal nanoparticle loading for a higher reaction extent. Of course, loading of $\mathrm{Fe}$ nanoparticles in the membrane depends on the extent of functionalization with PAA. Our earlier reported research with PAA functionalized microfiltration membranes extensively investigated the impact of functionalization conditions on the loading of nanoparticles. ${ }^{32,33}$ Furthermore, carboxylic functionalities in the membrane can potentially immobilize the leached Fe and also provides an opportunity to re-functionalize the membrane matrix with nanoparticles by repeating ion-exchange cycles. Higher nanoparticle loading could be achieved by increasing the proportion of PAA in the nanocomposite domain of the membrane. However, its influence on the perm-selectivity of the membrane will require further investigation.

Reactivity of the nanoparticles with persulfate depends on the oxidation state of iron in the nanoparticles. Minerals/oxides containing iron in $\mathrm{Fe}$ (II) form, such as magnetite, are reported to have high reactivity compared to iron in Fe(III) form, such as ferric oxide. $^{34}$ Therefore, characterization was extended to determine the crystalline structure of nanoparticles. TEM characterization was performed for nanoparticles synthesized in the solution phase in the absence of the membrane (Fig. 3ae). A thin oxide layer was observed on the surface of the 

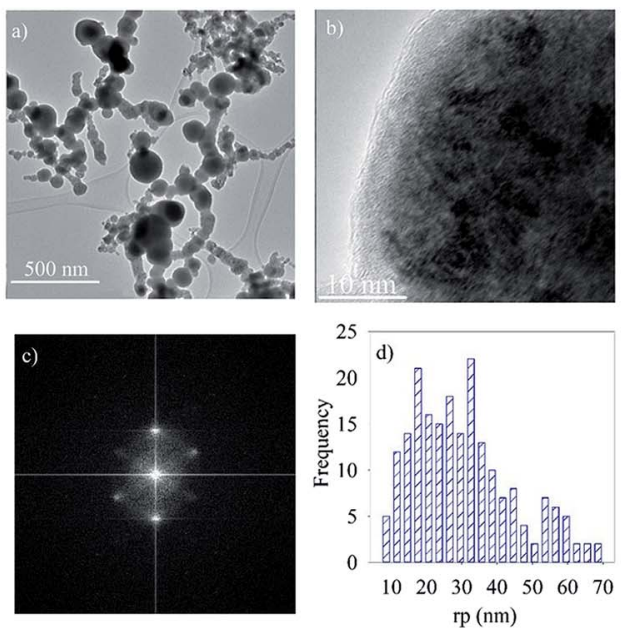
$\mathrm{rp}(\mathrm{nm})$

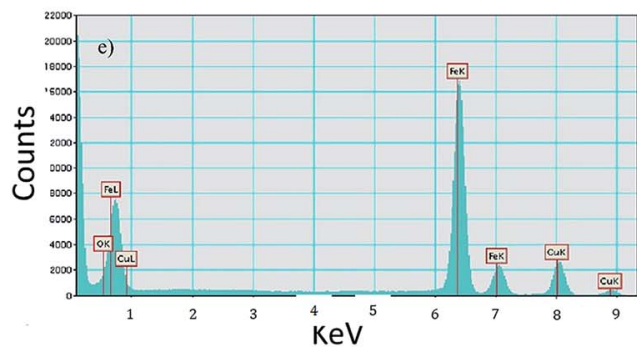

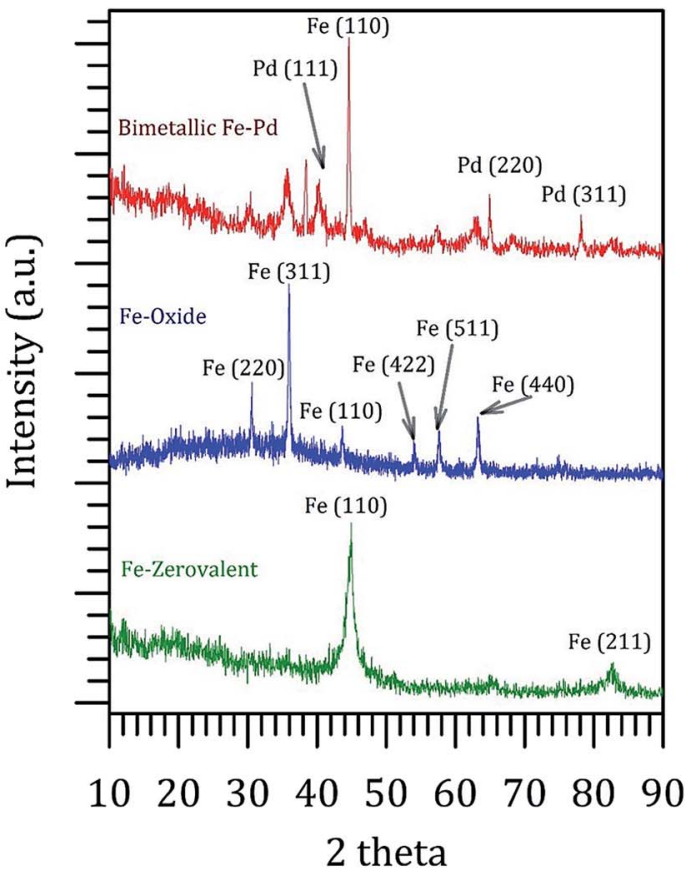

2 theta

Fig. 3 Characterization of solution-phase synthesized iron nanoparticles by transmission electron microscopy (TEM) and X-ray diffraction (XRD). Nanoparticles were synthesized in solution phase from ferrous ion precursors in the absence of membranes. (a) TEM image, (b) high-resolution TEM image, (c) SAED pattern, (d) size distribution, (e) EDS and (f) XRD pattern: (bottom) synthesized zerovalent iron nanoparticles, (middle) nanoparticles after oxidation persulfate oxidation reactions and (top) Fe-Pd bimetallic nanoparticles.

nanoparticles. The crystalline structures of the nanoparticles obtained using XRD were consistent with the literature..$^{35,36}$ Synthesized zerovalent Fe nanoparticles had a dominant peak at $2 \theta=45^{\circ}$ corresponding to $\mathrm{Fe}(110)$ (Fig. 3f). Post-coating of nanoparticles with palladium showed diffraction peaks corresponding to $\operatorname{Pd}(111)$ and $\operatorname{Pd}(311)$ at $38^{\circ}$ and $76^{\circ}$, respectively. The BET analysis of the nanoparticles showed a type II isotherm with a specific surface area of $8 \mathrm{~m}^{2} \mathrm{~g}^{-1}$ (ESI Fig. S6†). Also, it is worth mentioning that we have used our previously published protocol for the nanoparticle synthesis, and detailed characterizations of the bimetallic Fe-Pd nanoparticles could be found elsewhere. ${ }^{37}$ Characterization of nanoparticles (synthesized in solution phase) using TEM, XRD and BET thus suggested that $\mathrm{Pd}$ is indeed coated on the Fe-nanoparticles, and these nanoparticles have a non-porous spherical geometry.

During the synthesis of the membranes, $\mathrm{GO}$ was reduced to improve stability. Membranes were incubated at $90{ }^{\circ} \mathrm{C}$ for 24 hours. After reduction, a significant loss of free water and hydroxyl functionalities for GO was observed. A decline in oxygen content, an increase in hydrophobicity, and a decrease in interlayer spacing were observed. Characterization of the GO during thermal conditions is reported in our earlier publication. ${ }^{38}$

\subsection{Water permeability and separation performance}

Separation performance of rGO in loose nanofiltration regime was desired from the nanocomposite membranes, as the removal of larger organics before activation of persulfate in the membrane domain would prevent side reactions of these organics with sulfate-free radicals. The performance of the nanocomposite membrane was investigated to measure pure water permeability, salt retention and humic acids (model compound for NOM). rGO membranes are negatively charged loose nanofiltration membranes with a sizeexclusion cutoff around $1.2 \mathrm{~nm}$ in an aqueous media and can partially retain salts. ${ }^{39,40}$ In this study, performance was tested in two cross-flow cells operated in a parallel configuration, one containing the rGO membrane (rGO loading: $120 \mathrm{mg} \mathrm{m}^{-2}$ ) and other containing the nanocomposite rGO membrane.

Nanocomposite rGO and rGO membranes exhibited a pure water permeability of 1.9 and $2.3 \mathrm{LMH} \mathrm{bar}{ }^{-1}$, respectively, for TMP up to 7 bar (Fig. 4a). Initially, we aimed at quantifying the change, if any, in the size-based exclusion performance of the rGO membranes after the introduction of nanoparticles in the membrane matrix. Neutral red dye (size: $1.2 \mathrm{~nm}, \mathrm{M}$ Wt: $289 \mathrm{~g}$ $\mathrm{mol}^{-1}$ ) was used as a probe molecule to check for any microscopic defects introduced in the membranes during synthesis. Graphene oxide membranes are known to have a high retention of neutral red (over 90\%). ${ }^{41}$ Both, rGO and nanocomposite (with metal nanoparticles) rGO membranes exhibited high retention of neutral red. It is interesting to note that the permselectivity of the membrane was not significantly affected by the incorporation of nanoparticles in the membrane domain, suggesting the incorporated functionalized domain didn't add 

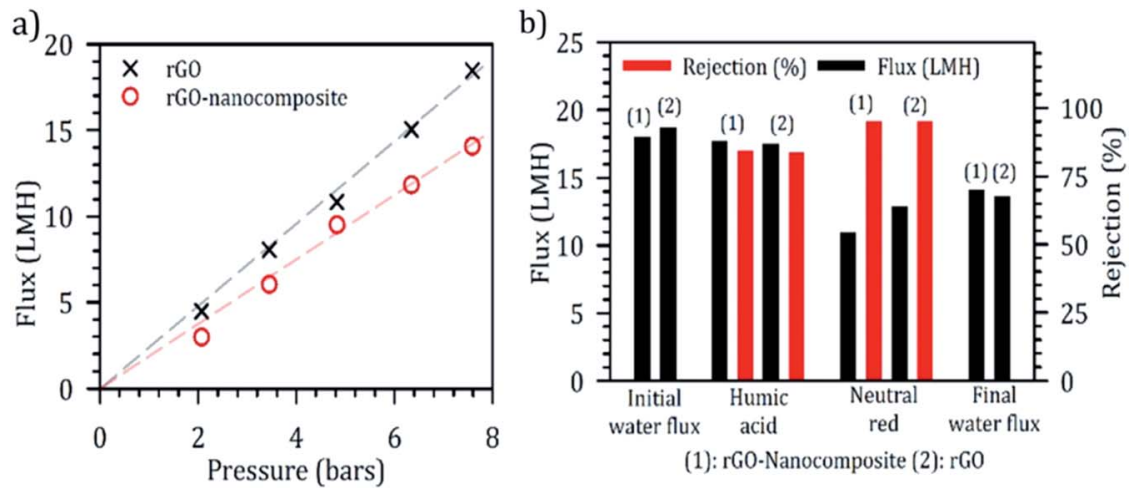

Fig. 4 Performance of $\mathrm{rGO}$ and $\mathrm{rGO}$ nanocomposite membranes. (a) Permeability, and (b) humic acid and neutral red retention by the membranes. Humic acid and neutral red retention was measured at 6.9 bar operating pressure gradient and a feed concentration of 100 ppm and 50 ppm, respectively.

significant resistance for the transport of molecules across the membrane (Fig. 4b). During the study, both membranes showed significant decay in the flux of both the membranes, around $60 \%$, during the filtration of neutral red. Average flux recovery of around $75 \%$ after rinsing the membranes with pure water was observed.

GO membranes are known to partially retain salts owing to the presence of negatively charged carboxylate groups in the confined interlayer spacing. ${ }^{\mathbf{4 2}}$ Salt retention by the membranes was evaluated using $\mathrm{Na}_{2} \mathrm{SO}_{4}, \mathrm{NaCl}, \mathrm{MgSO}_{4}$, and $\mathrm{MgCl}_{2}$ for a feed concentration of $7 \mathrm{mM}$ and at a TMP of 7 bar. The corresponding rejections of the four salts by nanocomposite rGO membrane were $55 \%, 30 \%, 25 \%$, and $5 \%$, respectively, and by rGO membrane were $56 \%, 47 \%, 43 \%$, and $16 \%$, respectively (Fig. S13 $\dagger$ ). The observed trend in salt retention indicates salt exclusion by rGO and nanocomposite rGO membranes is charge driven, as the rejection of $\mathrm{MgCl}_{2}$ was lower than of $\mathrm{NaCl}$, and the rejection of $\mathrm{MgSO}_{4}$ was lower than $\mathrm{Na}_{2} \mathrm{SO}_{4}$. Donnan exclusion is reported as a major contributor to the underlying exclusion mechanism compared to size exclusion. ${ }^{43}$ GO sheets tend to swell in contact in water, and the effective interlayer spacing is larger than hydration radii of ion. ${ }^{44}$ Therefore, steric hindrance has a significantly lower contribution to the overall exclusion mechanism of ions. Lower salt retention by rGO membranes can be advantageous in applications requiring organic removal from water containing high salt concentrations or where removal of minerals from water is not desired, as one would expect a lesser extent of fouling caused by hardness precipitation and lower osmotic pressure gradient during the treatment of such water streams by rGO membranes. ${ }^{45}$

Next, we tested the retention of NOM by rGO membranes using $100 \mathrm{ppm}$ humic acids (model compound) as feed in a cross-flow mode of operation. Both membranes showed high retention of humic acids, rejecting around $80 \%$ of the feed (Fig. 4b). Partial permeation of humic acids through the membranes can be attributed to the polydispersity of humic acids. The flux of the membranes declined by $10 \%$ after 6 hours of operation during the experiment. Humic acids are anionic macromolecules and are expected to be retained by
GO membranes through steric hindrance and charge repulsion.

\subsection{Reactivity performance}

3.3.1 Persulfate decomposition. A persulfate mediated pathway was employed for reactive removal of organic contaminants. A persulfate mediated pathway has advantages over the one mediated by hydrogen peroxide, such as longer half time of the sulfate-free radicals compared to hydroxyl radicals, selectively higher reaction rates with electron-rich contaminants, less severe scavenging effect of persulfate on sulfate radicals compared to that of hydrogen peroxide on hydroxyl radicals, and enhanced degradation rates at elevated temperatures. ${ }^{46,47}$

An initial set of experiments were conducted with different Fe-based nanoparticles to quantify the removal efficiency. Persulfate activation by zerovalent Fe (1:1 molar ratio) was tested by reusing the nanoparticles over two cycles using a batch mode experiment. For the first run, persulfate decomposed at a rapid rate with all persulfate being consumed within a reaction time of sixty minutes (ESI Fig. S7†). The second cycle of persulfate activation, with used Fe from the first run, showed no conversion of persulfate suggesting all Fe was converted to ferric oxide, which was later confirmed by XRD (Fig. 3f).

The reactive properties of Fe-based nanoparticles for persulfate activation are found to improve by doping $\mathrm{Fe}$ nanoparticles with group 10-11 elements, such as $\mathrm{Cu}, \mathrm{Ni}$, and $\mathrm{Pd}^{48}$ The pathway for the reaction is reported to proceed through the generation of sulfate-free radicals. ${ }^{49}$ Therefore, an initial set of experiments were conducted with different Fe-based nanoparticles to quantify the persulfate decomposition efficiency. Pd doped Fe nanoparticles were also investigated for the persulfate activation. Reactive performance for persulfate decomposition by zerovalent $\mathrm{Fe}$ and $\mathrm{Fe} / \mathrm{Pd}$ bimetallic (90:10 Fe : Pd atomic ratio) particles (1 : 1 molar ratio) was evaluated over four cycles of 24 hours in a batch-mode operation. Nanoparticles were reused during repeated cycles of the experiment. For zerovalent Fe nanoparticles, high persulfate decomposition occurred initially, which dropped substantially $(<5 \%)$ for the subsequent cycles (ESI Fig. S8†). However, Fe/Pd bimetallic nanoparticles 
retained its reactivity during the experiment and had an average decomposition efficiency of $40 \%$ over four cycles. Considering the better reactive performance shown by bimetallic particles, Fe nanoparticles embedded in the membranes were post coated with Pd.

Reactive performance of the nanocomposite membranes was tested by passing $2 \mathrm{mM}$ persulfate solution through the membrane in a dead-end mode of operation at two TMPs ( $\mathrm{pH} 7$ and $23{ }^{\circ} \mathrm{C}$ ), over 17 hours of operation. Passing persulfate solution through the membrane has an advantage of improved mass transfer coefficients, as the reactants are convectively driven to the nanoparticles embedded in the membrane pores. Initially, filtration was operated at TMP of 0.4 bar, for which more than $95 \%$ conversion of persulfate was observed. For subsequent filtration operation at a TMP of 1.4 bar (one-third the residence time at $0.4 \mathrm{bar}$ ), one would expect about $85 \%$ steady state conversion, but the drop to $26 \%$ indicated lower reactivity of oxidized iron/Pd nanoparticles (Fig. 5). Corresponding flux at the two investigated TMP were 2 and 7 LMH. Leaching of iron during the experiment was also monitored by measuring the concentration of dissolved iron in the permeate by inductively coupled plasma. Iron loss due to leaching was around $8 \%$ during the experiment. For the lower operating flux, the residence time of the reactant in the reactive domain is higher, and therefore, a greater extent of reaction is expected.

3.3.2 Stability of rGO membranes. Naturally, the stability of the rGO membrane in an aqueous and oxidative environment is vital for the application of these membranes. The reduction of GO is advantageous in this case as it imparts stability in two ways. First, reduction of GO enhances the pi-pi stacking of the deposited sheets, preventing them from dislodging off the substrate.$^{50}$ Second, reduction of GO is essential for improving the resistance of these materials towards oxidizing radicals. Functionalities present on GO, such as hydroxyl and epoxy are more susceptible to cleave from the backbone. Furthermore,

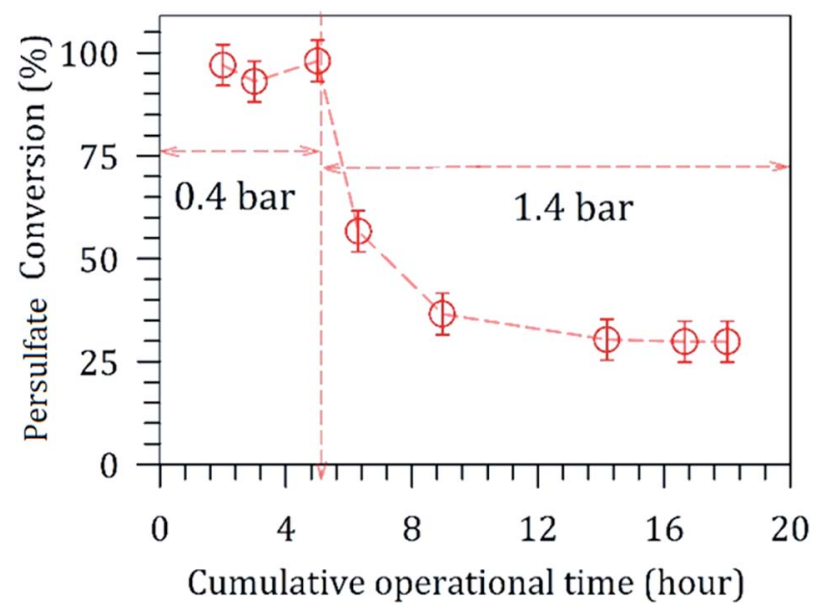

Fig. 5 Activation of persulfate by nanocomposite GO membranes Persulfate decomposition was conducted in a dead-end mode of operation. [Persulfate_feed] $=2 \mathrm{mM}, \mathrm{pH}=7$, temp. $=23^{\circ} \mathrm{C}$. The $x$-axis shows the total cumulative operational time ( $>17$ hours) during a study conducted over a span of 17 days. owing to the high electron density of these electron-rich functionalities, they are susceptible to the attack of electrophilic sulfate free radicals. Furthermore, the activation energy for the removal of these functionalities is expected to decrease with the increasing extent of oxidation of GO. Partial removal of these functionalities by reduction of GO is therefore important. In this study, the rGO membranes showed a negligible flux change during the experiment represented by Fig. 5, suggesting stable performance of the membranes under an oxidative environment (ESI Fig. S9†). An additional experiment was performed by treating $\mathrm{rGO}$ with a persulfate solution at an elevated temperature of $50{ }^{\circ} \mathrm{C}$. rGO samples after 24 hours and 72 hours of exposure were characterized by Raman and FTIR spectroscopy (ESI Fig. S10 and S11†). During the experiment complete decomposition of persulfate occurred; however, no apparent changes in either of the spectra were observed, suggesting no significant introduction of defects or change in functionalities in rGO films during persulfate exposure.

3.3.3 Oxidative removal of trichloroethylene. The residence time of the reactant in the membrane domain is typically short (of the order of few seconds) for a reasonable flux. ${ }^{51}$ It, therefore, necessitates fast reaction kinetics to achieve a greater extent of organic contaminant oxidation. To establish the kinetics of the reaction, persulfate mediated oxidation of a saturated solution of TCE was conducted. The saturated solution of TCE comprised of a liquid TCE droplet in equilibrium with water ( $2 \mathrm{ml}$ TCE per liter water). Chloride produced during the reaction was monitored to determine the extent of reaction using a chloride probe. Oxidation of TCE by sulfate free radicals generates chloride as byproduct, and in an ideal situation, this reaction should result in complete mineralization of TCE which would yield three moles of chloride for one mole of TCE. ${ }^{34}$ Before every run, persulfate $(7.2 \mathrm{mM})$ was added to the saturated TCE solution and chloride formation was monitored for 30 minutes. During this phase, no significant amount of chloride was formed, suggesting relatively slower kinetics of TCE

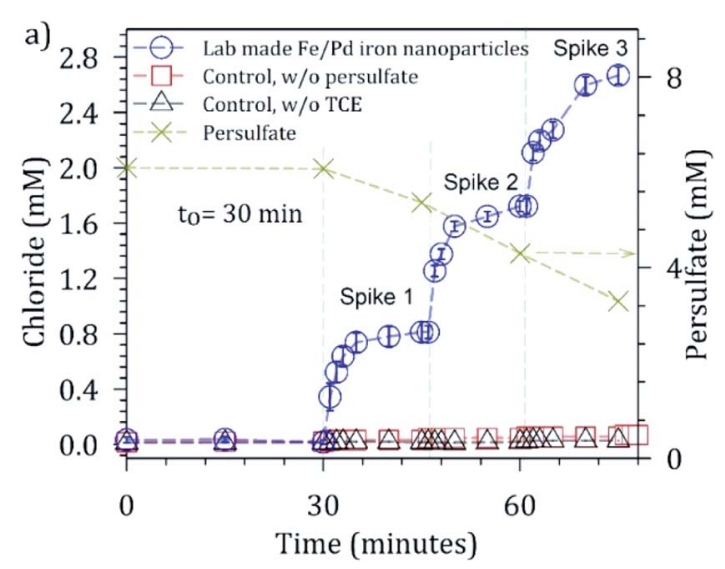

Fig. 6 Trichloroethylene decomposition by persulfate mediated oxidative pathway in the solution phase. Solution phase decomposition conducted with a saturated TCE-water phase solution (2000 ppm). Generated chloride was monitored after spiking solution containing $7 \mathrm{mM}$ persulfate with 20 ppm Fe/Pd nanoparticles. 
decomposition by persulfate itself. The saturated solution of TCE containing persulfate was then spiked with Fe/Pd nanoparticles $(20 \mathrm{ppm})$. A rapid increase in chloride concentration was observed, as shown in Fig. 6. Three replicates were performed, each run consisting of three successive spikes of nanoparticles. The reaction produced $0.4 \mathrm{mM}$ of chloride within 60 seconds of reaction and a total of $0.8 \mathrm{mM}$ chloride by 5 minutes after spiking $20 \mathrm{ppm}$ nanoparticles. Eventually, the reaction slowed dramatically owing to the consumption of $\mathrm{Fe}$ (II) sites.

After ensuring fast kinetics, TCE oxidation experiments were conducted with the nanocomposite rGO membranes by passing solutions through the membrane under pressure gradient. TCE solution $(0.3 \mathrm{mM})$ was passed through the membranes in the presence of $2 \mathrm{mM}$ persulfate solution at TMPs (transmembrane pressure) of $0.5,1$, and 2 bar in a dead-end mode of operation. The oxidation extent of TCE was determined by measuring the concentration of TCE on both the feed and permeate sides. Respective TCE conversion achieved at the three TMPs were 61, 52 and $27 \%$, respectively (Fig. 7). The ratio of the amount of chloride produced/maximum possible chloride for TCE oxidation was $0.63,0.70$, and 0.70 , respectively. An additional control run performed with TCE solution in the absence of persulfate showed a loss of up to $14 \%$ of TCE and was attributed to adsorption and vapor losses. The nanocomposite membranes thus offer an opportunity to achieve a controlled extent of reaction by regulating transmembrane pressure, and thus, residence time.

One of the advantages of employing persulfate mediated oxidation reactions is a significant enhancement in the reaction rates at elevated temperatures due to thermally activated fission of peroxy linkage. The effect of temperature on removal efficiencies of TCE $(0.7 \mathrm{mM}$ feed $)$ was investigated by conducting a solution-phase oxidation reaction at $4{ }^{\circ} \mathrm{C}, 23{ }^{\circ} \mathrm{C}$ and $40{ }^{\circ} \mathrm{C}$ in the presence of nanocomposite membranes $\left(0.5 \mathrm{~cm}^{2}\right)$ and $4 \mathrm{mM}$ persulfate in $20 \mathrm{ml}$ glass vials (Fig. 8). During the runs, vials

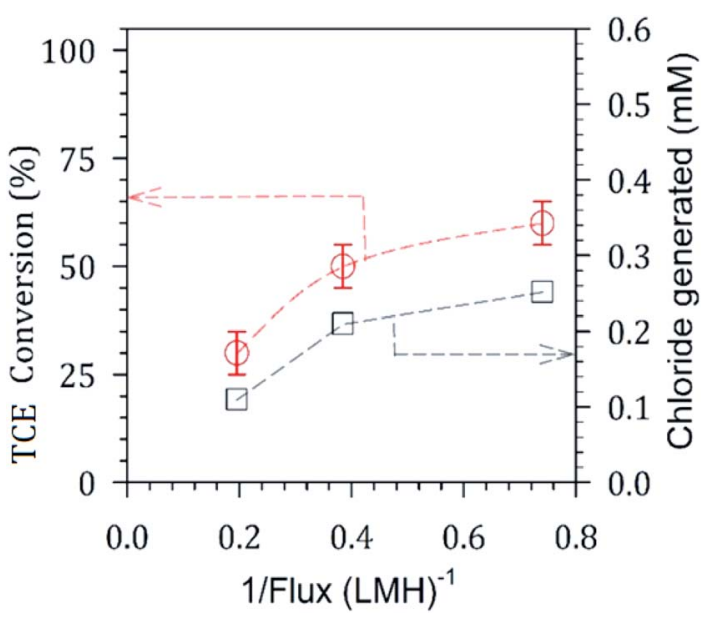

Fig. 7 Trichloroethylene decomposition by persulfate mediated oxidative pathway initiated by the nanocomposite membrane. Oxidation was conducted with $0.2 \mathrm{mM}$ TCE in the presence of $2 \mathrm{mM}$ persulfate at $\mathrm{pH} 7$ and $23^{\circ} \mathrm{C}$.
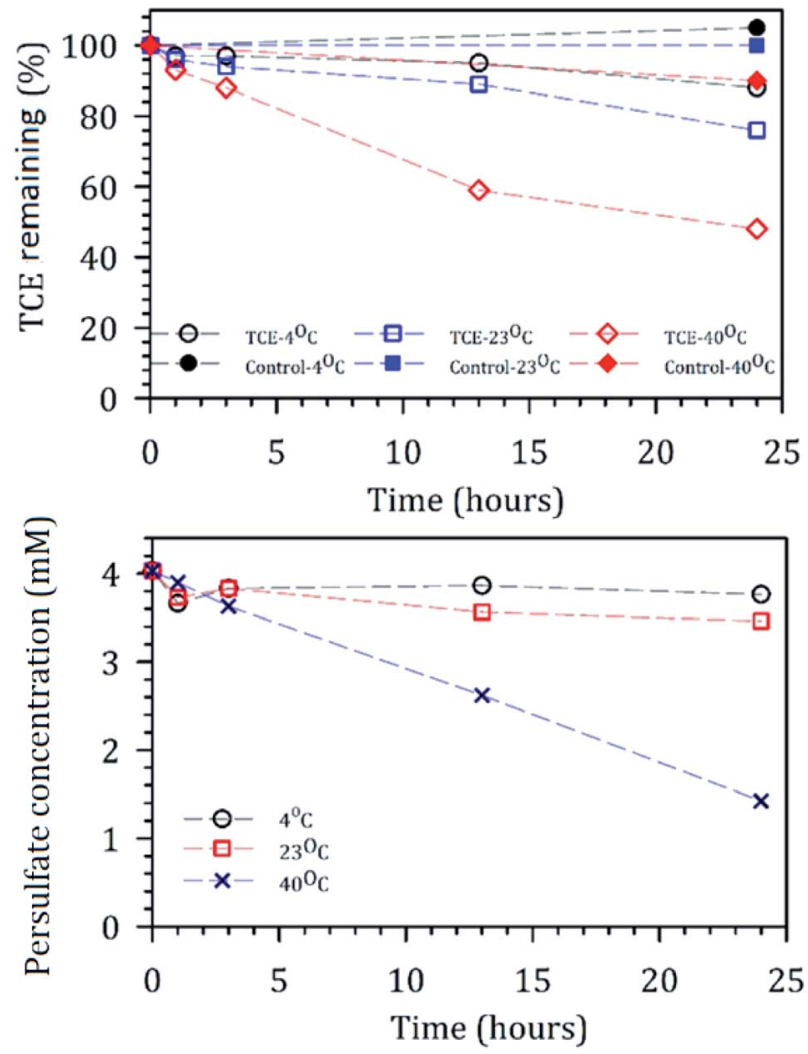

Fig. 8 Persulfate mediated oxidation of TCE in the presence of the nanocomposite membranes (soaking mode, no convective flow) at different temperatures. TCE_feed: $0.7 \mathrm{mM}$, persulfate: $4 \mathrm{mM}$. Decomposition experiments were conducted in $20 \mathrm{ml}$ glass vials with no headspace. $0.5 \mathrm{~cm}^{2}$ of membrane samples were used for oxidation reactions.

were taken from the sets at different time intervals, and concentration of persulfate and TCE were measured. Control samples showed a loss of $<1 \%, 4 \%$, and $10 \%$ at $4{ }^{\circ} \mathrm{C}, 21{ }^{\circ} \mathrm{C}$, and $40{ }^{\circ} \mathrm{C}$ in the absence of persulfate. Corresponding decomposition of persulfate at the three temperatures after 24 hours of reaction time was $5 \%, 10 \%$, and $65 \%$, respectively, clearly signifying the role of temperature in activation of persulfate. TCE degradation after 24 hours at corresponding temperatures were $10 \%, 22 \%$, and $54 \%$, respectively. Lower conversion (compared to convective flow, Fig. 7) would be expected due to internal mass transfer limitation. Thus, our results suggest elevated temperatures can also be effectively used in enhancing persulfate mediated TCE removal efficiency.

The oxidation of organic contaminants for a sulfate-free radical-mediated pathway occurs via the formation of organic radical cation through the electron transfer from organic compounds to the sulfate radical. ${ }^{52}$ Subsequent hydrolysis of the organic radical cations results in the formation of oxygenated products. To analyze the intermediates of persulfate mediated TCE oxidation, solution-phase oxidation of $0.7 \mathrm{mM}$ TCE was conducted in the presence of persulfate $(5 \mathrm{mM})$ and nanoparticles (100 ppm). For a reaction time of 1 hour, 30\% conversion of TCE was observed. The reaction mixture was then 
reacted with hexyl alcohol in the presence of $\mathrm{H}_{2} \mathrm{SO}_{4}$ at $60 \%$ to esterify the acidic reaction intermediates. The GC-MS analysis of the esterified reaction mixture suggested the presence of chloro-acetic acid as one of the products (ESI Fig. S12†). Similar products are reported for hydroxyl radical-mediated oxidation of TCE. ${ }^{46}$

\section{Conclusion}

In summary, we demonstrated the synthesis of nanocomposite reactive rGO membranes with capabilities for separation in loose nanofiltration regime and to initiate advanced oxidation reactions for the removal of trace organic contaminants from water. rGO domain was functionalized with cross-linked polyacrylic acid using an LBL assembly, which enabled in situ synthesis of nanoparticles. TEM characterization of the film showed a high concentration of iron nanoparticles $(12 \% \mathrm{Fe}$, $0.6 \% \mathrm{Pd}$ ) in the PAA functionalized domain. The functionalized membranes retained the separation performance of rGO layer, exhibiting $80 \%$ humic acid and partial salt rejection, which is important in reducing the number of unwanted side reactions. rGO layer after incorporation of nanoparticles retained its selectivity towards retaining a neutral red probe molecule (size: $1.2 \mathrm{~nm}$, rejection $>90 \%$ ). The membranes effectively decomposed persulfate and degraded up to $61 \%$ TCE (at $23{ }^{\circ} \mathrm{C}$ ), generating up to $70 \%$ of the theoretical maximum chloride, in a convective mode of operation. Obviously, one can easily increase the conversion by controlling residence time through the membrane pores and also by multiple passes or running at higher temperatures. Increase in temperatures enhanced persulfate mediated TCE oxidation (in batch mode) from $<5 \%$ at $4{ }^{\circ} \mathrm{C}$ to $54 \%$ at $40{ }^{\circ} \mathrm{C}$. Derivatization of the products after oxidation of TCE showed the presence of chloro-acetic acids as one of the by-products. The reactive membranes synthesized in this study can potentially serve applications in the removal of TCE and other smaller organic contaminants through an oxidative pathway and exclusion of interfering larger organics by charge and size-based exclusion.

\section{Conflicts of interest}

The authors declare no conflict of interest.

\section{Acknowledgements}

This research was funded by the NIEHS Superfund program (Grant no. PE42ES007380) and NSF-EPSCOR (Grant no: 1355438). Dr Nicolas Briot and Dr Dali Qian assisted with the sample preparation for TEM and TEM characterization, respectively. Analysis of Trichloroethylene was conducted at the Environmental Research and Training Laboratory at University of Kentucky. Dr Andrew Colburn, Hongyi Wan, and Saiful Islam provided valuable insights at various stages of the manuscript.

\section{References}

1 M. R. Chowdhury, J. Steffes, B. D. Huey and J. R. Mccutcheon, 3D Printed Polyamide Membranes for Desalination, Science, 2018, 686, 682-686.

2 A. Deshmukh, C. Boo, V. Karanikola, S. Lin, A. P. Straub, T. Tong, D. M. Warsinger and M. Elimelech, Membrane Distillation at the Water-Energy Nexus: Limits, Opportunities, and Challenges, Energy Environ. Sci., 2018, 11(5), 1177-1196, DOI: 10.1039/c8ee00291f.

3 S. O. Ganiyu, E. D. Van Hullebusch, M. Cretin, G. Esposito and M. A. Oturan, Coupling of Membrane Filtration and Advanced Oxidation Processes for Removal of Pharmaceutical Residues: A Critical Review, Sep. Purif. Technol., 2015, 156, 891-914, DOI: 10.1016/j.seppur.2015.09.059.

4 J. R. Werber, C. O. Osuji and M. Elimelech, Materials for Next-Generation Desalination and Water Purification Membranes, Nat. Rev. Mater., 2016, 1, 16018.

5 H. N. Sandefur, M. Asgharpour, J. Mariott, E. Gottberg, J. Vaden, M. Matlock and J. Hestekin, Recovery of Nutrients from Swine Wastewater Using Ultrafiltration: Applications for Microalgae Cultivation in Photobioreactors, Ecol. Eng., 2016, 94, 75-81.

6 A. Aher, J. Papp, A. Colburn, H. Wan, E. Hatakeyama, P. Prakash, B. Weaver and D. Bhattacharyya, Naphthenic Acids Removal from High TDS Produced Water by Persulfate Mediated Iron Oxide Functionalized Catalytic Membrane, and by Nanofiltration, Chem. Eng. J., 2017, 327, 573-583, DOI: 10.1016/j.cej.2017.06.128.

7 B. der Bruggen and C. Vandecasteele, Removal of Pollutants from Surface Water and Groundwater by Nanofiltration: Overview of Possible Applications in the Drinking Water Industry, Environ. Pollut., 2003, 122(3), 435-445.

8 M. Sun, I. Zucker, D. M. Davenport, X. Zhou, J. Qu and M. Elimelech, Reactive, Self-Cleaning Ultrafiltration Membrane Functionalized with Iron Oxychloride Nanocatalysts, Environ. Sci. Technol., 2018, 52(15), 86748683, DOI: 10.1021/acs.est.8b01916.

9 S. R. Lewis, S. Datta, M. Gui, E. L. Coker, F. E. Huggins, S. Daunert, L. Bachas and D. Bhattacharyya, Reactive Nanostructured Membranes for Water Purification, Proc. Natl. Acad. Sci. U. S. A., 2011, 108(21), 8577-8582, DOI: 10.1073/pnas.1101144108.

10 S. Z. Islam, A. Reed, D. Y. Kim and S. E. Rankin, $\mathrm{N}_{2} /$ Ar Plasma Induced Doping of Ordered Mesoporous $\mathrm{TiO}_{2}$ Thin Films for Visible Light Active Photocatalysis, Microporous Mesoporous Mater., 2016, 220, 120-128.

11 L. Huang, J. Chen, T. Gao, M. Zhang, Y. Li, L. Dai, L. Qu and G. Shi, Reduced Graphene Oxide Membranes for Ultrafast Organic Solvent Nanofiltration, Adv. Mater., 2016, 28(39), 8669-8674, DOI: 10.1002/adma.201601606.

12 R. K. Joshi, S. Alwarappan, M. Yoshimura, V. Sahajwalla and Y. Nishina, Graphene Oxide: The New Membrane Material, Appl. Mater. Today, 2015, 1(1), 1-12, DOI: 10.1016/ j.apmt.2015.06.002. 
13 Y. Liu, L. Yu, C. N. Ong and J. Xie, Nitrogen-Doped Graphene Nanosheets as Reactive Water Purification Membranes, Nano Res., 2016, 9(7), 1983-1993, DOI: 10.1007/s12274-0161089-7.

14 D. E. Jiang, B. G. Sumpter and S. Dai, Unique Chemical Reactivity of a Graphene Nanoribbon's Zigzag Edge, J. Chem. Phys., 2007, 126(13), 1-24, DOI: 10.1063/1.2715558.

15 H. Sun, S. Liu, G. Zhou, H. M. Ang, M. O. Tadé and S. Wang, Reduced Graphene Oxide for Catalytic Oxidation of Aqueous Organic Pollutants, ACS Appl. Mater. Interfaces, 2012, 4(10), 5466-5471, DOI: 10.1021/am301372d.

16 Z. Song, M. Wang, Z. Wang, Y. Wang, R. Li, Y. Zhang, C. Liu, Y. Liu, B. Xu and F. Qi, Insights into Heteroatom-Doped Graphene for Catalytic Ozonation: Active Centers, Reactive Oxygen Species Evolution, and Catalytic Mechanism, Environ. Sci. Technol., 2019, acs.est.9b01361, DOI: 10.1021/ acs.est.9b01361.

17 J. Liang, Y. Jiao, M. Jaroniec and S. Z. Qiao, Sulfur and Nitrogen Dual-Doped Mesoporous Graphene Electrocatalyst for Oxygen Reduction with Synergistically Enhanced Performance, Angew. Chem., Int. Ed., 2012, 51(46), 11496-11500, DOI: 10.1002/anie.201206720.

18 W. Tian, H. Zhang, X. Duan, H. Sun, M. O. Tade, H. M. Ang and S. Wang, Nitrogen- and Sulfur-Codoped Hierarchically Porous Carbon for Adsorptive and Oxidative Removal of Pharmaceutical Contaminants, ACS Appl. Mater. Interfaces, 2016, 8(11), 7184-7193, DOI: 10.1021/acsami.6b01748.

19 X. Wang, Y. Qin, L. Zhu and H. Tang, Nitrogen-Doped Reduced Graphene Oxide as a Bifunctional Material for Removing Bisphenols: Synergistic Effect between Adsorption and Catalysis, Environ. Sci. Technol., 2015, 49(11), 6855-6864, DOI: 10.1021/acs.est.5b01059.

20 K. Ullah, S. Ye, L. Zhu, Z. D. Meng, S. Sarkar and W. C. Oh, Microwave Assisted Synthesis of a Noble Metal-Graphene Hybrid Photocatalyst for High Efficient Decomposition of Organic Dyes under Visible Light, Mater. Sci. Eng., B, 2014, 180(1), 20-26, DOI: 10.1016/j.mseb.2013.10.014.

21 J. Yan, W. Gao, M. Dong, L. Han, L. Qian, C. P. Nathanail and M. Chen, Degradation of Trichloroethylene by Activated Persulfate Using a Reduced Graphene Oxide Supported Magnetite Nanoparticle, Chem. Eng. J., 2016, 295, 309-316, DOI: 10.1016/j.cej.2016.01.085.

22 X. Huang, X. Qi, F. Boey and H. Zhang, Graphene-Based Composites, Chem. Soc. Rev., 2012, 41(2), 666-686, DOI: 10.1039/c1cs15078b.

23 A. Ahmad, X. Gu, L. Li, S. Lv, Y. Xu and X. Guo, Efficient Degradation of Trichloroethylene in Water Using Persulfate Activated by Reduced Graphene Oxide-Iron Nanocomposite, Environ. Sci. Pollut. Res., 2015, 22(22), 17876-17885, DOI: 10.1007/s11356-015-5034-1.

24 M. Pedrosa, G. Drazic, P. B. Tavares, J. L. Figueiredo and A. M. T. Silva, Metal-Free Graphene-Based Catalytic Membrane for Degradation of Organic Contaminants by Persulfate Activation, Chem. Eng. J., 2019, 369, 223-232, DOI: 10.1016/j.cej.2019.02.211.

25 Y. Gao, M. Hu and B. Mi, Membrane Surface Modification with $\mathrm{TiO}_{2}$-Graphene Oxide for Enhanced Photocatalytic
Performance, J. Membr. Sci., 2014, 455, 349-356, DOI: 10.1016/j.memsci.2014.01.011.

26 H. Zhang, Y. Zhu, J. Long, Z. Ding, R. Yuan, Z. Li and C. Xu, In Situ Construction of Layered Graphene-Based Nanofiltration Membranes with Interlayer Photocatalytic Purification Function and Their Application for Water Treatment, Environ. Sci.: Nano, 2019, 2195-2202, DOI: 10.1039/c9en00351g.

27 A. Soroush, W. Ma, Y. Silvino and M. S. Rahaman, Surface Modification of Thin Film Composite Forward Osmosis Membrane by Silver-Decorated Graphene-Oxide Nanosheets, Environ. Sci.: Nano, 2015, 2(4), 395-405, DOI: 10.1039/c5en00086f.

28 Y. Jiang, J. E. Goodwill, J. E. Tobiason and D. A. Reckhow, Impacts of Ferrate Oxidation on Natural Organic Matter and Disinfection Byproduct Precursors, Water Res., 2016, 96, 114-125.

29 P. Xie, W. Liu, J. Zou, S. Yue, W. Liu, J. Zou and S. Yue, Impact of UV/Persulfate Pretreatment on the Formation of Disinfection Byproducts during Subsequent Chlorination of Natural Organic Matter, Chem. Eng. J., 2015, 269, 203-211.

30 J. J. Song, Y. Huang, S. W. Nam, M. Yu, J. Heo, N. Her, J. R. V. Flora and Y. Yoon, Ultrathin Graphene Oxide Membranes for the Removal of Humic Acid, Sep. Purif. Technol., 2015, 144, 162-167, DOI: 10.1016/ j.seppur.2015.02.032.

31 C. Liang, C. F. Huang, N. Mohanty and R. M. Kurakalva, A Rapid Spectrophotometric Determination of Persulfate Anion in ISCO, Chemosphere, 2008, 73(9), 1540-1543, DOI: 10.1016/j.chemosphere.2008.08.043.

32 M. Gui, J. K. Papp, A. S. Colburn, N. D. Meeks, B. Weaver, I. Wilf and D. Bhattacharyya, Engineered Iron/Iron Oxide Functionalized Membranes for Selenium and Other Toxic Metal Removal from Power Plant Scrubber Water, J. Membr. Sci., 2015, 488, 79-91, DOI: 10.1016/ j.memsci.2015.03.089.

33 M. S. Islam, S. Hernandez, H. Wan, L. Ormsbee and D. Bhattacharyya, Role of Membrane Pore Polymerization Conditions for PH Responsive Behavior, Catalytic Metal Nanoparticle Synthesis, and PCB Degradation, J. Membr. Sci., 2018, 555, 348-361, DOI: 10.1016/j.memsci.2018.03.060.

34 M. Gui, V. Smuleac, L. E. Ormsbee, D. L. Sedlak and D. Bhattacharyya, Iron Oxide Nanoparticle Synthesis in Aqueous and Membrane Systems for Oxidative Degradation of Trichloroethylene from Water, J. Nanopart. Res., 2012, 14(5), 861-877.

35 S. R. Kanel, B. Manning, L. Charlet and H. Choi, Removal of Arsenic(III) from Groundwater by Nanoscale Zero-Valent Iron, Environ. Sci. Technol., 2005, 39(5), 1291-1298.

36 R. K. Petla, S. Vivekanandhan, M. Misra, A. K. Mohanty and N. Satyanarayana, Soybean (Glycine Max) Leaf Extract Based Green Synthesis of Palladium Nanoparticles, J. Biomater. Nanobiotechnol., 2012, 3(1), 14-19.

37 H. Wan, M. S. Islam, N. J. Briot, M. Schnobrich, L. Pacholik, L. Ormsbee and D. Bhattacharyya, Pd/Fe Nanoparticle Integrated PMAA-PVDF Membranes for Chloro-Organic 
Remediation from Synthetic and Site Groundwater, $J$. Membr. Sci., 2020, 594, 117454.

38 A. Aher, R. Sarma, M. Crocker and D. Bhattacharyya, Selective Molecular Separation of Lignin Model Compounds by Reduced Graphene Oxide Membranes from Solvent-Water Mixture, Sep. Purif. Technol., 2020, 230, 115865, DOI: 10.1016/j.seppur.2019.115865.

39 A. Akbari, P. Sheath, S. T. Martin, D. B. Shinde, M. Shaibani, P. C. Banerjee, R. Tkacz, D. Bhattacharyya and M. Majumder, Large-Area Graphene-Based Nanofiltration Membranes by Shear Alignment of Discotic Nematic Liquid Crystals of Graphene Oxide, Nat. Commun., 2016, 7, 10891, DOI: 10.1038/ncomms10891.

40 Y. Yuan, X. Gao, Y. Wei, X. Wang, J. Wang, Y. Zhang and C. Gao, Enhanced Desalination Performance of Carboxyl Functionalized Graphene Oxide Nanofiltration Membranes, Desalination, 2017, 405, 29-39, DOI: 10.1016/ j.desal.2016.11.024.

41 A. Akbari, S. Meragawi, S. Martin, B. Corry, E. Shamsaei, C. D. Easton, D. Bhattacharyya and M. Majumder, Solvent Transport Behavior of Shear Aligned Graphene Oxide Membranes and Implications in Organic Solvent Nanofiltration, ACS Appl. Mater. Interfaces, 2017, acsami.7b11777, DOI: 10.1021/acsami.7b11777.

42 E. Yang, M. H. Ham, H. B. Park, C. M. Kim, J. ho Song and I. S. Kim, Tunable Semi-Permeability of Graphene-Based Membranes by Adjusting Reduction Degree of Laminar Graphene Oxide Layer, J. Membr. Sci., 2018, 547, 73-79, DOI: 10.1016/j.memsci.2017.10.039.

43 Y. Han, Z. Xu and C. Gao, Ultrathin Graphene Nanofiltration Membrane for Water Purification, Adv. Funct. Mater., 2013, 23(29), 3693-3700, DOI: 10.1002/adfm.201202601.

44 B. Lian, S. De Luca, Y. You, S. Alwarappan, M. Yoshimura, V. Sahajwalla, S. C. Smith, G. Leslie and R. K. Joshi, Extraordinary Water Adsorption Characteristics of Graphene Oxide, Chem. Sci., 2018, 9(22), 5106-5111, DOI: 10.1039/c8sc00545a.
45 Y. A. Le Gouellec and M. Elimelech, Calcium Sulfate (Gypsum) Scaling in Nanofiltration of Agricultural Drainage Water, J. Membr. Sci., 2002, 205(1-2), 279-291.

46 S. Huling and B. Pivetz, Engineering Issue: In-Situ Chemical Oxidation, United States Environmental Protection Agency, 2006, pp. 1-60.

47 S. Ko, M. Crimi, B. K. Marvin, V. Holmes and S. G. Huling, Comparative Study on Oxidative Treatments of NAPL Containing Chlorinated Ethanes and Ethenes Using Hydrogen Peroxide and Persulfate in Soils, J. Environ. Manage., 2012, 108, 42-48, DOI: 10.1016/ j.jenvman.2012.04.034.

48 M. A. Al-Shamsi, N. R. Thomson and S. P. Forsey, Iron Based Bimetallic Nanoparticles to Activate Peroxygens, Chem. Eng. J., 2013, 232, 555-563.

49 Y. Feng, P. H. Lee, D. Wu and K. Shih, Surface-Bound Sulfate Radical-Dominated Degradation of 1,4-Dioxane by AluminaSupported Palladium $\left(\mathrm{Pd} / \mathrm{Al}_{2} \mathrm{O}_{3}\right) \quad$ Catalyzed Peroxymonosulfate, Water Res., 2017, 120, 12-21, DOI: 10.1016/j.watres.2017.04.070.

50 A. Aher, Y. Cai, M. Majumder and D. Bhattacharyya, Synthesis of Graphene Oxide Membranes and Their Behavior in Water and Isopropanol, Carbon, 2017, 116, 145-153.

51 H. Wan, N. J. Briot, A. Saad, L. Ormsbee and D. Bhattacharyya, Pore Functionalized PVDF Membranes with In-Situ Synthesized Metal Nanoparticles: Material Characterization, and Toxic Organic Degradation, $J$. Membr. Sci., 2017, 530, 147-157, DOI: 10.1016/ j.memsci.2017.02.021.

52 G. P. Anipsitakis, D. D. Dionysiou and M. A. Gonzalez, Cobalt-Mediated Activation of Peroxymonosulfate and Sulfate Radical Attack on Phenolic Compounds. Implications of Chloride Ions, Environ. Sci. Technol., 2006, 40(3), 1000-1007, DOI: 10.1021/es050634b. 